\title{
基于纳米银负载氧化石墨烯的新型聚乙烯复合材料
}

\author{
张 峰 ${ }^{1,2}$, 张凯立 ${ }^{1}$, 周明明 ${ }^{2}$, 陈 超 $^{3}$, 蔡志威 ${ }^{3}$, 魏国辉 ${ }^{4}$, \\ 姜兴茂 ${ }^{4,5}$, 张 诚 ${ }^{1}$, 劳伦 . 鲁尔曼 ${ }^{6}$, 吕耀康 ${ }^{1}$
}

(1. 浙江工业大学 化学工程学院, 杭州 310014; 2. 浙江大学医学院附属儿童医院, 杭州 $310052 ; 3$. 浙江省食品药 品检验研究院，杭州 310052；4. 常州英中纳米科技有限公司，常州 213000；5. 武汉工程大学 化工与制药学院， 武汉 430205; 6. 斯特拉斯堡大学 化学研究所, 斯特拉斯堡 67081)

摘 要: 通过超声波辅助液相法将纳米银(AgNPs)与氧化石墨烯(GO)结合制得了一种新的负载纳米银的氧化石墨烯材 料 AgNPs@GO。分析表明在该材料中 $\mathrm{AgNPs}$ 主要被针接在 $\mathrm{GO}$ 片层的含氧基团和缺陷上, 部分 $\mathrm{Ag}$ 单质被氧化为 $\mathrm{Ag}^{+}$ 离子并有部分 GO 被还原。AgNPs@GO 能有效抑制铜绿假单胞菌生长, 其抑菌能力显著强于 AgNPs 和 GO。将 $\mathrm{AgNPs@GO}$ 作为添加剂引入聚乙烯 $(\mathrm{PE})$ 基体，进一步制备了新型的 AgNPs@GO 掺杂 PE 复合材料 $0.48 w t \%-A g N P s @ G O / P E$, 相比 PE 和 AgNPs 掺杂 PE 复合材料, $0.48 \mathrm{wt} \%-\mathrm{AgNPs} @ \mathrm{GO} / \mathrm{PE}$ 具有更好的抑菌能力和更强的阻隔水蒸气性能, 并 且在水和乙醇溶液中都具有较好的耐溶出性能。

关 键 词: 纳米银; 氧化石墨烯; 铜绿假单胞菌; 聚乙烯; 抑菌; 阻隔水蒸气性能

中图分类号: O614 文献标识码: A

\section{A New Polyethylene Composite Material Based on Nano Silver Particels Loaded Graphene Oxide}

\begin{abstract}
ZHANG Feng $^{1,2}$, ZHANG Kai-Li ${ }^{1}$, ZHOU Ming-Ming ${ }^{2}$, CHEN Chao ${ }^{3}$, CAI Zhi-Wei ${ }^{3}$, WEI Guo-Hui ${ }^{4}$, JIANG Xing-Mao $^{4,5}$, ZHANG Cheng ${ }^{1}$, Laurent RUHLMANN ${ }^{6}$, LÜ Yao-Kang ${ }^{1}$

(1. School of Chemical Engineering, Zhejiang University of Technology, Hangzhou 310014, China; 2. The Children's Hospital, Zhejiang University School of Medicine, Hangzhou 310052, China; 3. Zhejiang Institute for Food and Drug Control, Hangzhou, 310052, China; 4. Changzhou Yingzhong Nano Technology Co., Ltd, Changzhou 213000, China; 5. School of Chemical Engineering \& Pharmacy, Wuhan Institute of Technology, Wuhan 430205, China; 6. Institut de Chimie, Université de Strasbourg, Strasbourg 67081, France)
\end{abstract}

\begin{abstract}
A new nano silver particels loaded graphene oxide (AgNPs@GO) was obtained through ultrasonic assisted liquid phase method. In AgNPs@GO, nano silver particels (AgNPs) are mainly anchored at oxygen containing groups and defects of graphene oxide (GO) sheets, part of GO sheets are reduced and some $\operatorname{Ag}(0)$ atoms have been oxidized to $\mathrm{Ag}^{+}$ions. AgNPs@GO exhibits significantly stronger ability for inhibiting Pseudomonas aeruginosa than that of AgNPs and GO. Therefore, AgNPs@GO was further introduced to polyethylene (PE) matrix as an additive to prepare a new composite material 0.48wt\%-AgNPs@GO/PE. Compare with bare PE and AgNPs doped PE composites, 0.48wt\%-AgNPs@GO/PE not only has a better antibacterial ability and stronger water vapor barrier property, but also dissolves out less nonvolatile substance than PE in water and ethanol solution.
\end{abstract}

Key words: nano silver particels; graphene oxide; pseudomonas aeruginosa; polyethylene; antibacterial ability; water vapor barrier property

收稿日期: 2018-08-09; 收到修改稿日期：2018-09-04

基金项目: 国家自然科学基金(21501148); 浙江省药品接触材料质量控制研究重点实验室开放基金; 浙江省自然科学基金 (LY19B01000)

National Natural Science Foundation of China (21501148); Open Funding from Zhejiang Provincial Key Laboratory for Quality Control of Drug Contact Materials; Zhejiang Provincial Natural Science Foundation (LY19B01000)

作者简介: 张 峰(1980-), 男, 副主任医师. E-mail: zfwl@zju.edu.cn

通讯作者：吕耀康, 副研究员. E-mail: yaokanglv@zjut.edu.cn 
随着经济高速发展以及人民生活质量的提高, 对口服药剂以及方便食品的需求量不断增加, 与之 紧密相联的塑料包装材料也在向绿色、抗菌、高阻 隔等方向发展 ${ }^{[1-2]}$ 。聚乙烯(Polyethylene, PE) 是目前 最常用的包装材料之一，但普通的 PE 材料对氧气、 水蒸气的阻隔性能不佳, 易被细菌污染, 不利于食 品和药品的保存 ${ }^{[3]}$ 。

纳米银(Silver nanoparticles, AgNPs) 是一种抗菌 谱广、抗菌效能久、热稳定性好、生物安全性高的 纳米抗菌材料 ${ }^{[4-5]} 。 \mathrm{AgNPs}$ 可以作为功能化添加剂对 PE 等包装材料进行改性, 制备抗菌包装材料, SanchezValdes $^{[6]}$ 将 AgNPs 沉积在线性低密度聚乙烯/环烯烃 共聚物(LLDPE/COC)共混物挤出膜表面, 获得了对 尼日尔曲霉属真菌有良好抑菌活性的包装膜。李振 兴等 ${ }^{[7]}$ 制备了 $\mathrm{AgNPs}$ 质量分数为 $1 \%$ 和 $2 \%$ 的抗菌活 性 PE 薄膜, 可延长虾仁在冷藏过程中的保质期。但 是 AgNPs 的表面作用能很强, 纳米粒子之间极易团 聚，导致制备的 PE复合材料容易产生缺陷，影响其 阻隔水蒸气性能等物理化学性能。因此, 研究和开 发 AgNPs 均匀分散的 PE复合材料, 在不降低 PE 材 料物理化学性能的前提下赋予 PE 复合材料高效的 抗菌能力, 具有重要的意义。

氧化石墨烯(Graphene Oxide, GO)具有优异的 杀菌活性及温和的细胞毒性, 也可以作为高效抗菌 剂使用 ${ }^{[8]} ; \mathrm{GO}$ 独特的平面结构使含 $\mathrm{GO}$ 的复合材料 具有一定的阻隔小分子透过的能力, 而 GO 边缘丰 富的亲水官能团, 使其能均匀地分散在水等极性溶 液中，并易于被其它基团修饰或负载 ${ }^{[9-11]}$, 最近的 研究表明, 将金属纳米粒子与 GO 结合, 可能产生 协同作用，获得具备优异性能的新材料 ${ }^{[12-14]}$ 。本研 究通过超声波辅助液相复合法成功获得了纳米银负 载氧化石墨烯材料 AgNPs@GO, 实验发现该材料 抑制铜绿假单胞菌的能力显著强于 AgNPs 和 $\mathrm{GO}$;
进一步将AgNPs@GO 作为添加剂引入 $\mathrm{PE}$ 基体, 制 备新型的 $\mathrm{AgNPs} @ \mathrm{GO}$ 掺杂聚乙烯复合薄片材料, 并研究了该复合材料的抗菌性能、耐溶出性能和阻 隔水蒸气性能。

\section{1 实验方法}

\subsection{AgNPs@GO 的制备}

$\mathrm{GO}$ 采用改良的 Hummers 法 ${ }^{[15]}$ 制备(补充材料 1), AgNPs 的制备参照发明专利“制备单分散超细颗粒 的方法”公开的制备方法 ${ }^{[16]}$ ，由常州英中纳米科技 有限公司提供(产品号 YZN-Y400, AgNPs 含量为 $0.4 \mathrm{wt} \%$ ), 本研究所用的其它试剂均为分析纯(补充 材料 2)。如图 1 所示, 将 $128 \mathrm{mg}$ 的 $\mathrm{GO}$ 粉末和 $100 \mathrm{~mL}$ AgNPs 溶胶加入 $100 \mathrm{~mL}$ 的乙醇中, 使用频率为 $40 \mathrm{KHz}$ 的超声波发生器在 $40{ }^{\circ} \mathrm{C}$ 超声波振荡 $2 \mathrm{~h}$, 得 到澄清的 AgNPs@GO 溶胶, 在 $60{ }^{\circ} \mathrm{C}$ 真空环境干燥 $12 \mathrm{~h}$, 得到 $\mathrm{AgNPs} @ \mathrm{GO}$ 固体粉末, 其中 $\mathrm{AgNPs}$ : GO 的质量比为 $3: 1$ 。

\section{$1.2 A g N P s @ G O$ 掺杂聚乙烯复合材料的制备}

将 AgNPs@GO 复合材料与 PE 母粒按质量比 AgNPs@GO : PE = 0.48: 99.52 进行搅拌共混, 在 $60{ }^{\circ} \mathrm{C}$ 真空环境干燥, 并置于双棍筒开炼机中, 在 $160{ }^{\circ} \mathrm{C}$ 下进 行混炼，再使用平板硫化仪在 $160{ }^{\circ} \mathrm{C}$ 的温度下制备得 到厚度为 $1.0 \mathrm{~mm}$ 的 $\mathrm{AgNPs} @ \mathrm{GO}$ 掺杂聚乙烯复合材料 薄片，该新材料被命名为 $0.48 \mathrm{wt} \%-\mathrm{AgNPs} @ \mathrm{GO} / \mathrm{PE}$ 。

将 $\mathrm{AgNPs}$ 和 $\mathrm{PE}$ 母粒分别按照质量比为 $\mathrm{AgNPs}$ : $\mathrm{PE}=0.48: 99.52$ 和 $2.88: 97.12$ 进行摚拌共混, 在 $60{ }^{\circ} \mathrm{C}$ 真空环境干燥，采用上述相同制备方法得到不 同 AgNPs 含量的复合材料薄片, 依次命名为 $0.48 \mathrm{wt} \%$-AgNPs/PE，2.88wt\%-AgNPs/PE，并将它们 与 $0.48 \mathrm{wt} \%-\mathrm{AgNPs} @ \mathrm{GO} / \mathrm{PE}$ 的性能进行比较。
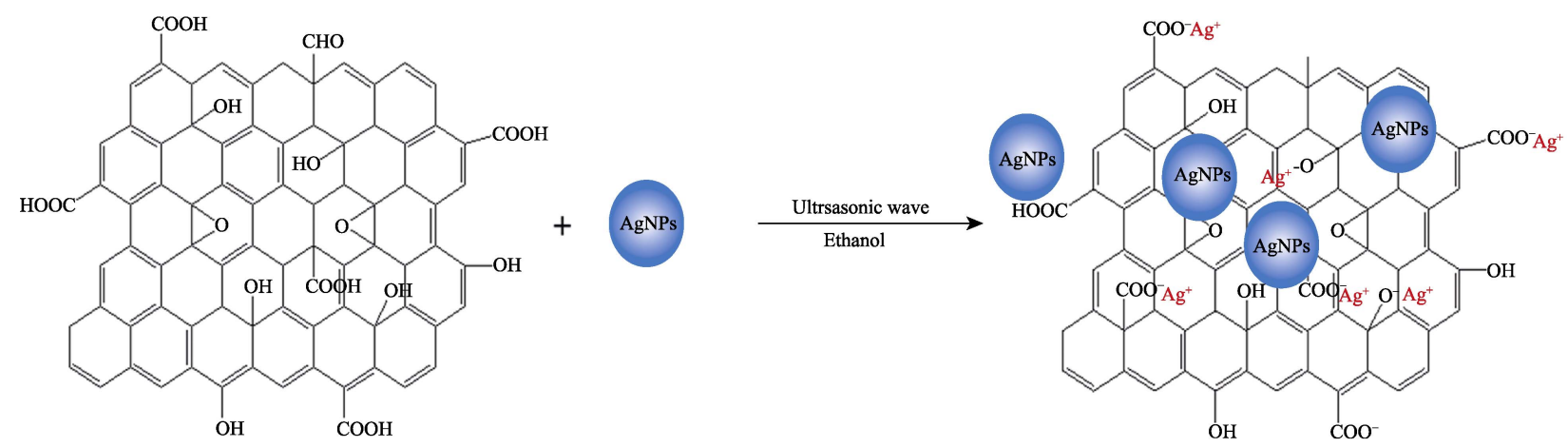

图 1 AgNPs@GO 的制备流程图

Fig. 1 Schematic illustration for the preparation of AgNPs@GO 


\section{3 结构和形貌的表征}

透射电子显微镜(TEM)数据采集自 FEI 公司 Tecnai G2 F30 S-Twin 透射电镜; X 射线光电子能谱 (XPS)和俄歇电子能谱(AES)采用岛津 AXIS Kratos Ultra DLD 多功能光电子能谱仪测试(补充材料 4); 红外光谱(IR)分析采用赛默飞公司的 Nicolet 6700 spectrometer 型傅里叶转换红外光谱仪补充材料 5); 紫外-可见光谱(UV-Vis)分析采用岛津紫外-可见光 光度计 UV-2700; X 射线粉末衍射(pXRD)分析采用 Bruker D8 衍射仪(补充材料 6)。

\section{4 抗菌性能研究}

选用铜绿假单胞菌标准菌株(ATCC27853, 购 自美国菌种收藏中心), 用纸片扩散法和直接接触 实验测试AgNPs@GO、GO 和 AgNPs 三种材料的抑 菌性能; 用直接接触实验测试 $0.48 \mathrm{wt} \%-\mathrm{AgNPs} / \mathrm{PE}$ 、 $2.88 \mathrm{wt} \%-\mathrm{AgNPs} / \mathrm{PE}$ 和 $0.48 \mathrm{wt} \%-\mathrm{AgNPs} @ \mathrm{GO} / \mathrm{PE}$ 三 种材料的抑菌性能。将 $-80{ }^{\circ} \mathrm{C}$ 冻存的 ATCC27853 菌 株复苏, 在 $35{ }^{\circ} \mathrm{C}$ 培养 $24 \mathrm{~h}$ 后接种于哥伦比亚血琼 脂培养基上。

纸片扩散法实验步骤为: 挑取 ATCC27853 菌落, 用无菌生理盐水调制 $0.5 \mathrm{MCF}$ 左右的菌液 $5 \mathrm{~mL}$ 。用 无菌棉签蘸取上述 $0.5 \mathrm{MCF}$ 左右的菌液均匀涂布于 $\mathrm{MH}$ 培养基上, 之后将相同质量的AgNPs@GO、GO 和 AgNPs 三种溶胶涂布在直径为 $12.0 \mathrm{~mm}$ 的纸片上, 将纸片分别贴于培养基上, 然后将培养基放置于 $35{ }^{\circ} \mathrm{C}$ 培养箱中培养 $24 \mathrm{~h}$ 后取出。观察抑菌圈大小, 抑菌圈大小为抑制细菌生长的抑菌圈直径用游标卡尺 测量并记录。每个抑菌圈测量 3 次, 精确到 $0.01 \mathrm{~mm}$, 取平均值。

AgNPs@GO、GO 和 AgNPs 三种材料直接接触 实验的步骤为: 挑取 ATCC27853 菌落, 用无菌 LB 肉汤调制 $0.5 \mathrm{MCF}$ 左右的菌液 $4 \mathrm{~mL}$ 。取 4 个无菌试 管, 1 个试管内加入 $1 \mathrm{~mL}$ 菌液作为空白对照, 用上 述菌液分别将AgNPs@GO、GO 和 AgNPs 三种材料 配成终浓度为 $0.024 \mathrm{mg} / \mathrm{mL}$ 的溶液各 $1 \mathrm{~mL}$, 振荡试 管使材料分布均匀, 然后将试管放入 $35{ }^{\circ} \mathrm{C}$ 恒温箱 培养。用生物梅里埃的比浊仪分别在 $0 、 1 、 3 、 6$ 和 $24 \mathrm{~h}$ 测量各试管中 ATCC27853 菌液的浊度, 每 个试管测 3 次, 取其平均值; 分别在 $0 、 1 、 3 、 6$ 和 $24 \mathrm{~h}$ 时取菌液接种于营养琼脂平板上进行细菌培养 及计数。

$0.48 \mathrm{wt} \%-\mathrm{AgNPs} / \mathrm{PE} 、 2.88 \mathrm{wt} \%-\mathrm{AgNPs} / \mathrm{PE}$ 和 $0.48 \mathrm{wt} \%-\mathrm{AgNPs} @ \mathrm{GO} / \mathrm{PE}$ 三种材料的直接接触实验 的步骤为: 挑取 ATCC27853 菌落, 用无菌生理盐水
调制 $0.5 \mathrm{MCF}$ 左右的菌液 $5 \mathrm{~mL}$ 。取 4 个无菌试管, 每个试管内加入 $1 \mathrm{~mL}$ 上述配制的 $0.5 \mathrm{MCF}$ 左右的 $\mathrm{ATCC} 27853$ 菌液。分别将 $0.48 \mathrm{wt} \%-\mathrm{AgNPs} / \mathrm{PE}$ 、 $2.88 \mathrm{wt} \%-\mathrm{AgNPs} / \mathrm{PE}$ 和 $0.48 \mathrm{wt} \%-\mathrm{AgNPs} @ \mathrm{GO} / \mathrm{PE} 三$ 种材料各 $50 \mathrm{mg}$ 置于 3 个试管内, 振荡试管使材料 分布均匀, 另外 1 管不加任何材料作为阴性对照。 然后将试管放入 $35{ }^{\circ} \mathrm{C}$ 恒温箱培养, 用生物梅里埃 的比浊仪分别在 $1 、 3 、 6 、 24 、 48$ 和 $72 \mathrm{~h}$ 测量各试 管中 ATCC27853 菌液的浊度，每个试管测 3 次，取 其平均值。

\section{5 耐溶出性能和阻隔水蒸气性能研究}

溶出物实验参考《中华人民共和国药典》中对 塑料包装样品的溶出物实验的测试方法(补充材料 7 和 8$)$; 水蒸气透过率数据采集自 MOCON 公司 MCON Pematran-W3/33 水蒸气透过率测试仪。

\section{2 结果与讨论}

\subsection{AgNPs@GO 的结构与形貌}

如图 2 所示, AgNPs@GO 粉末呈棕黑色, 能够 被超声分散在水中形成淡棕色透明的 AgNPs@GO 溶胶。如图 S1 所示, AgNPs 溶胶的紫外吸收光谱在 $420 \mathrm{~nm}$ 附近显示出 $\mathrm{Ag}$ 单质的特征吸收峰, 而 $\mathrm{AgNPs} @ \mathrm{GO}$ 溶胶的紫外吸收光谱(图 S1)与 GO 溶胶 相似, 在 $300 \mathrm{~nm}$ 附近均出现了较强的吸收带, 这是 由 $\mathrm{GO}$ 碳骨架双键所连 $-\mathrm{C}=\mathrm{O}$ 上未成键的孤对电子 的 $\mathrm{n} \rightarrow \pi^{*}$ 跃迁产生的 $\mathrm{R}$ 带 ${ }^{[17]}$ 。AgNPs@GO 溶胶在 $400 \sim 600 \mathrm{~nm}$ 处的光吸收强度较相同浓度的 GO 溶胶 强, 这一现象可以归因于 AgNPs 与 GO 的结合。

图 3(a, b) 是 AgNPs@GO 的 TEM 照片。由图可见, AgNPs 与 $\mathrm{GO}$ 结合后主要负载在 $\mathrm{GO}$ 片层上, 并且基 本没有团聚。从 AgNPs@GO 的高分辨 TEM(HRTEM) 照片中可以观察到 AgNPs 晶粒的晶格条纹间距为
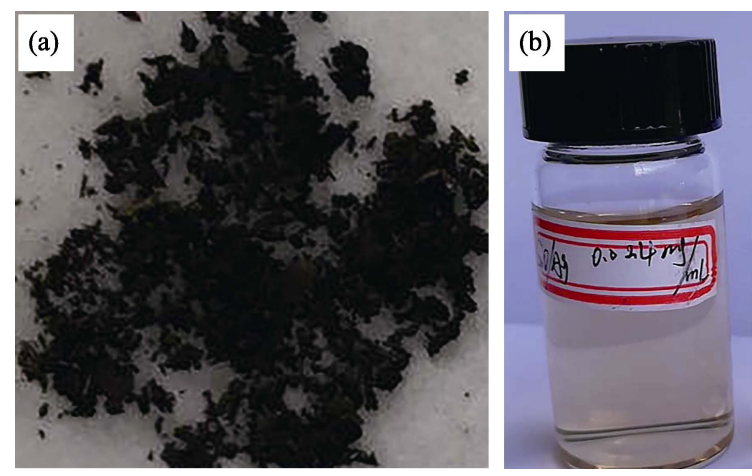

图 $2 A g N P s @ G O$ 粉末(a)和其溶胶(b)的照片

Fig. 2 Photographs of AgNPs@GO powder (a) and its sol (b) 

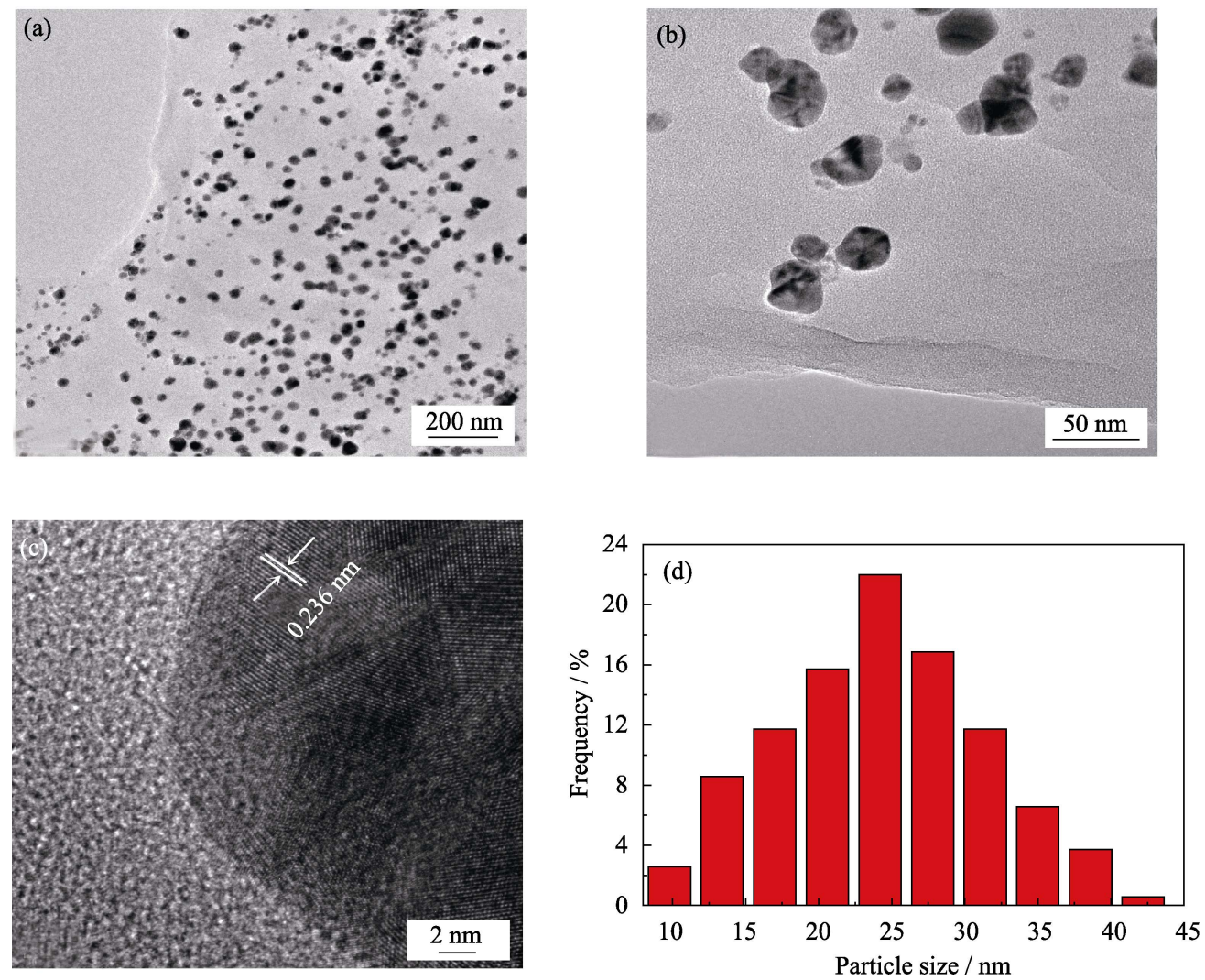

图 3 AgNPs@GO 的 TEM $(\mathrm{a}, \mathrm{b})$ 和 HRTEM(c)照片以及 AgNPs@GO 上负载的 AgNPs 粒径分布的统计结果 $(\mathrm{d})$

Fig. 3 TEM (a, b) and HRTEM (c) images of AgNPs@GO, and statistical result of the particle size distribution of AgNPs loaded on AgNPs@GO (d)

$0.236 \mathrm{~nm}$, 基本对应于立方 $\mathrm{Ag}$ 晶体的(111)晶面间 距(图 3(c))。如图 3(d)所示, 采用粒度分析软件 Nano Measurer1.2 对 AgNPs 的粒径分布进行统计分析, 分 析结果表明 AgNPs 的粒径分布主要集中在 20 30 nm 之间。

GO 和 AgNPs@GO 的 XRD 图谱如图 4(a)所示, $\mathrm{GO}$ 在 $2 \theta$ 约为 $10.3^{\circ}$ 附近有一个对应于其 $(001)$ 晶面

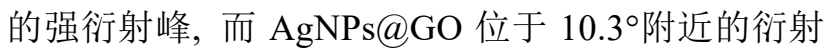
峰已基本消失, 同时其在 $23^{\circ}$ 附近对应于石墨烯 (002)晶面的漫峰显著增强, 说明在 AgNPs@GO 中, GO 已经被部分还原。此外, AgNPs@GO 在 $2 \theta$ 为 $38.1^{\circ} 、 44.3^{\circ} 、 64.4^{\circ}$ 和 $77.5^{\circ}$ 处的衍射峰分别对应于 面心立方结构的金属单质 $\operatorname{Ag}(J C P D S$ No.04-0783)的 (111)、(200)、(220)、(311)晶面, 通过 Debye-Scherrer 公式计算(补充材料 S4) 推测 $\mathrm{AgNPs} @ \mathrm{GO}$ 中纳米银晶 粒的平均粒径约为 $24 \mathrm{~nm}$, 这与 TEM 照片分析的结 果相一致。

图 4(b)为在动能 280 294 eV 区间, 对 GO 样品 窄区域高分辨扫描采集 $\mathrm{C} 1 \mathrm{~s}$ 的 XPS 图谱和应用拟合 软件 XPSpeak 4.1 对 C1s 谱峰进行分峰拟合的曲线。 从拟合的结果判断, 在 $\mathrm{GO}$ 中碳元素的主要存在形 式有 5 种, 分别为: $\mathrm{C}\left(\mathrm{sp}^{2}\right) 、 \mathrm{C}\left(\mathrm{sp}^{3}\right) 、 \mathrm{C}-\mathrm{O} 、 \mathrm{C}=\mathrm{O}$ 和
$\mathrm{O}-\mathrm{C}=\mathrm{O}$, 其对应的结合能分别为 $284 、 284.8 、 286.4$ 、 287.6 和 $288.9 \mathrm{eV}$ 。而对 $\mathrm{AgNPs} @ \mathrm{GO}$ 样品的 C1s 峰 的精细扫描及分峰拟合(图 4(c))表明, $\mathrm{C}-\mathrm{O}$ 和 $\mathrm{C}=\mathrm{O}$ 的结合能强度相对 GO 明显变小, 这一方面是由于 $\operatorname{AgNPs}$ 以 GO 的含氧官能团为结合点使其负载; 另 一方面原因是 AgNPs 在与 $\mathrm{GO}$ 结合时, 部分 $\mathrm{GO}$ 被 还原。图 4(d) 为在动能 526 538 eV 区间, 对 AgNPs@GO 样品的 O1s 峰的精细扫描及分峰拟合 的曲线。从拟合的结果判断, AgNPs@GO 中有大量 含氧官能团存在，氧的存在形式有 4 种: 与 AgNPs 连接的氧原子 $(530.2 \mathrm{eV})$ 、羟基氧 $(531.4 \mathrm{eV})$ 、羧基氧 $(532.4 \mathrm{eV})$ 以及样品中结合水中的氧原子 $(533.8 \mathrm{eV})$ 。

AgNPs@GO 样品的 Ag 3d 的XPS 窄区扫描图谱(图4(e)) 在 367.6 和 $373.8 \mathrm{eV}$ 处分别出现了 $\mathrm{Ag} 3 \mathrm{~d}_{5 / 2}$ 和 $\mathrm{Ag} 3 \mathrm{~d}_{3 / 2}$ 的特征峰, 而从 AgNPs@GO 的 AES 能谱(图 4(f)) 得出的最高峰的动能为 $351.8 \mathrm{eV}$ 。这一结果说明在 AgNPs@GO 中还存在 +1 价的 $\mathrm{Ag}^{+}$离子 ${ }^{[18]}$ 。

红外光谱分析也证实了上述结果(图 S3)。

目前已报道的纳米银/石墨烯复合材料的制备 方法大多是采用可溶性银盐为前驱体与石墨烯或 氧化石墨烯复合, 并采用还原性的化学试剂还原 $\mathrm{Ag}^{+}$离子制备复合材料 ${ }^{[19-20]}$ 。本研究通过微波辅助 

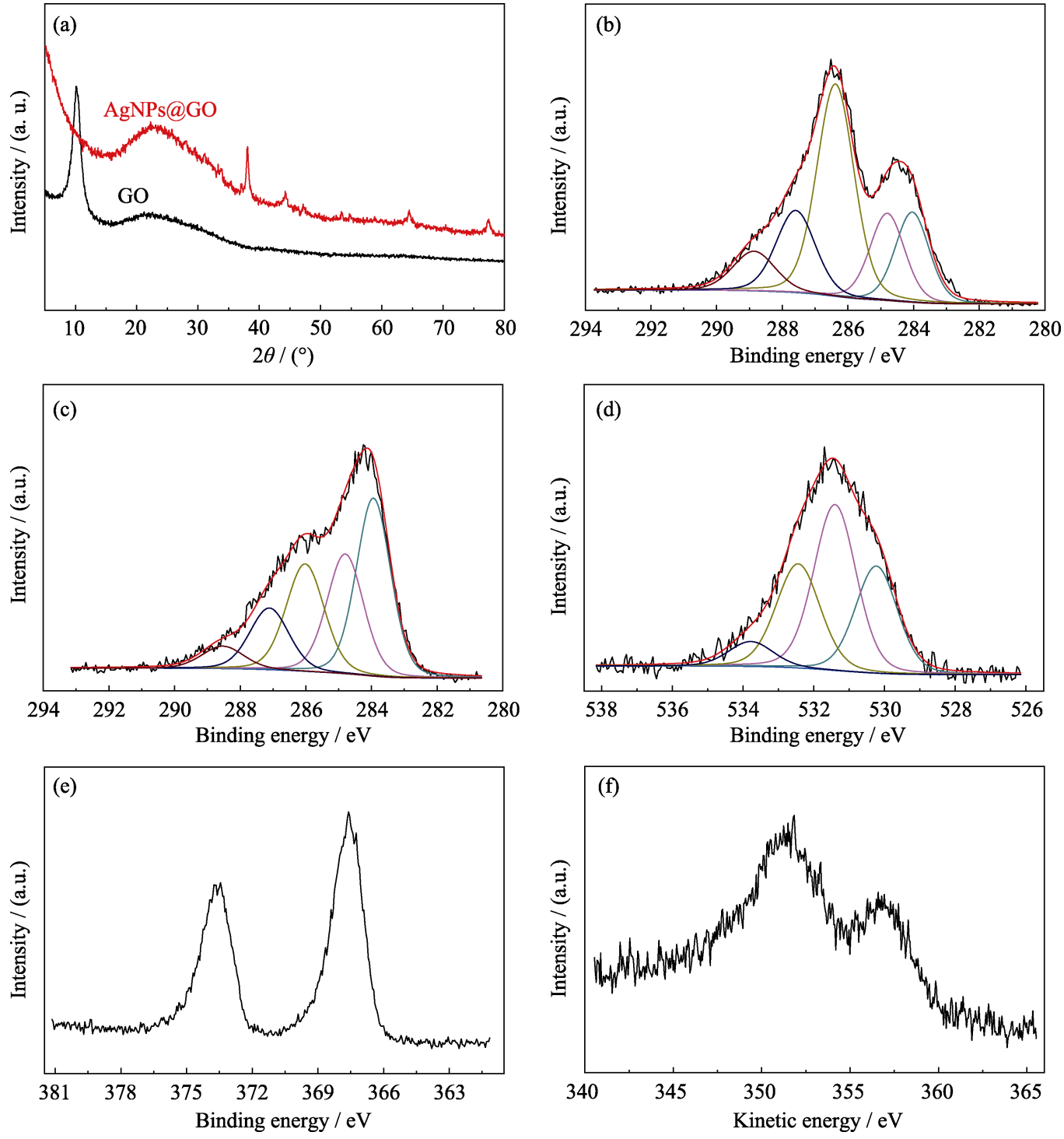

图 4 GO 和 AgNPs@GO 的 XRD 图谱(a); GO 的 XPS C1s 图谱(b); AgNP $@$ @ GO 的 $\mathrm{C} 1 \mathrm{~s}(\mathrm{c}) 、 \mathrm{O} 1 \mathrm{~s}(\mathrm{~d}) 、 \mathrm{Ag} 3 \mathrm{~d}(\mathrm{e})$ 的 XPS 图谱和 $\mathrm{Ag}(\mathrm{f})$ 的 AES 能谱

Fig. 4 XRD patterns of GO and AgNPs@GO(a); XPS C1s spectra of GO (b); XPS spectra C1s (c), O1s (d), Ag3d(e) of AgNPS@GO, and AES energy spectrum (f) of Ag

气溶胶技术获得单分散 AgNPs 颗粒和溶胶，并借 助超声波辐射压强产生的空化效应和扰动效应 增大 $\mathrm{GO}$ 和 $\mathrm{AgNPs}$ 溶胶粒子在溶剂中的运动频率 和速度, 使 $\mathrm{GO}$ 和 $\mathrm{AgNPs}$ 充分接触和结合, 制备 AgNPs@GO 溶胶。与现有方法相比，本方法更加 简便和绿色。

\section{$2.2 A g N P s @ G O$ 的抗菌性能}

如图 5 所示, AgNPs@GO 纸片的抑菌圈直径 $(26.85 \pm 0.35) \mathrm{mm}$ 明显大于 $\mathrm{GO}$ 纸片 $(23.10 \pm 0.10) \mathrm{mm}$ 和 AgNPs 纸片的 $(22.18 \pm 0.41) \mathrm{mm}$ 抑菌圈。从图 5 还可进一步发现 AgNPs@GO 抑菌圈周围梯度变 化较 AgNPs 和 GO 都更为明显, AgNPs@GO 周 围 $(17.30 \pm 0.09) \mathrm{mm}$ 范围内铜绿假单胞菌完全 抑制。
AgNPs@GO、GO 和 AgNPs 与铜绿假单胞菌的 直接接触实验结果如图 6 所示。1、3、6 和 $24 \mathrm{~h}$ 时取菌液接种于营养琼脂平板上进行细菌培养 (图 6(a))及计数结果(图 6(c)) 表明, AgNPs@GO 接触 组在这 4 个时间点琼脂平板上未见明显的铜绿假单 胞菌落, 而 $\mathrm{AgNPs}$ 和 $\mathrm{GO}$ 接触组在 $3 \mathrm{~h}$ 之后均出现 菌落生长, 可见AgNPs@GO 抑制铜绿假单胞菌生 长能力明显强于 $\mathrm{GO}$ 和 $\mathrm{AgNPs}$; 这一结果与比浊仪 在相同时间段测量各试管中菌液的浊度结果一致 (图 6(b))。

铜绿假单胞菌是医院内引起感染的常见致病菌 群之一，对临床多种抗生素产生一定的耐药性 ${ }^{[21]}$ 。 该细菌在口腔领面部手术后可引起伤口化脓性感染, 甚至导致败血症，危及患者的生命 ${ }^{[22]}$ 。以上实验结 

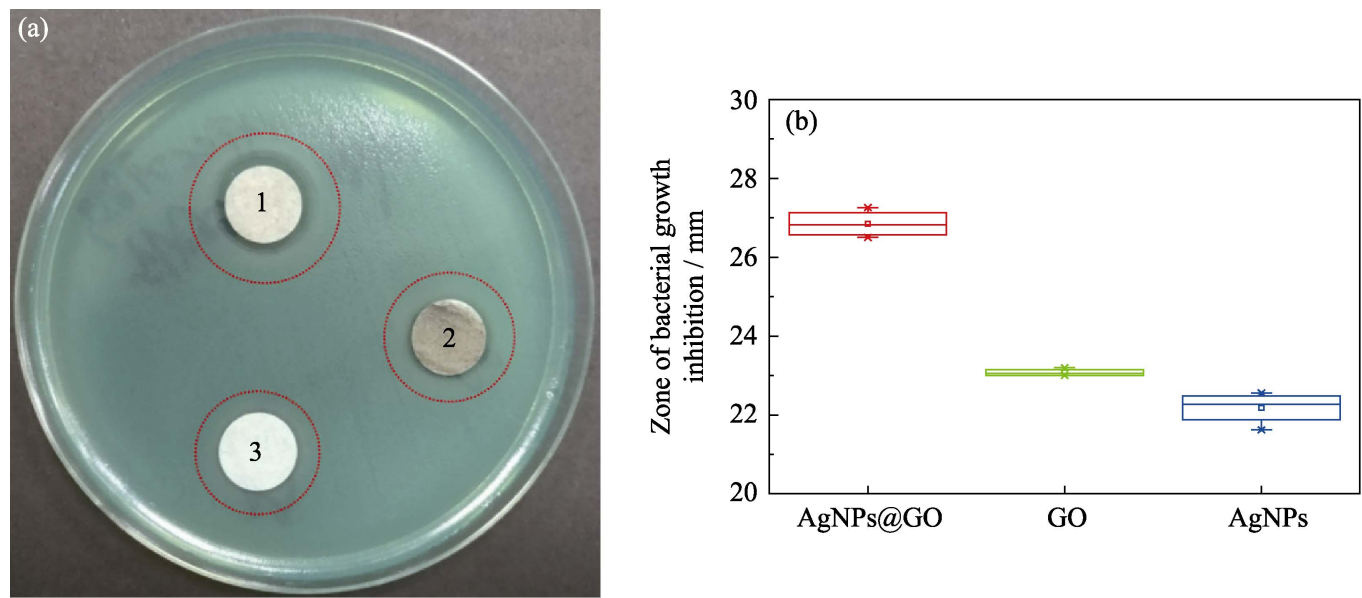

图 5 AgNPs@GO、GO 和 AgNPs 的铜绿假单胞菌的抑菌圈实验照片(a)((1)涂有 AgNPs@GO 的纸片,

(2)涂有 GO 的纸片, (3)涂有 AgNPs 的纸片)和抑菌圈实验数据结果图(b)

Fig. 5 Photograph of inhibition zone of Pseudomonas aeruginosa ((1) paper coated with AgNPs@GO, (2) paper coated with GO, (3) paper coated with AgNPs) (a) and results of experimental data of inhibition zone of AgNPs@GO, GO and AgNPs (b)
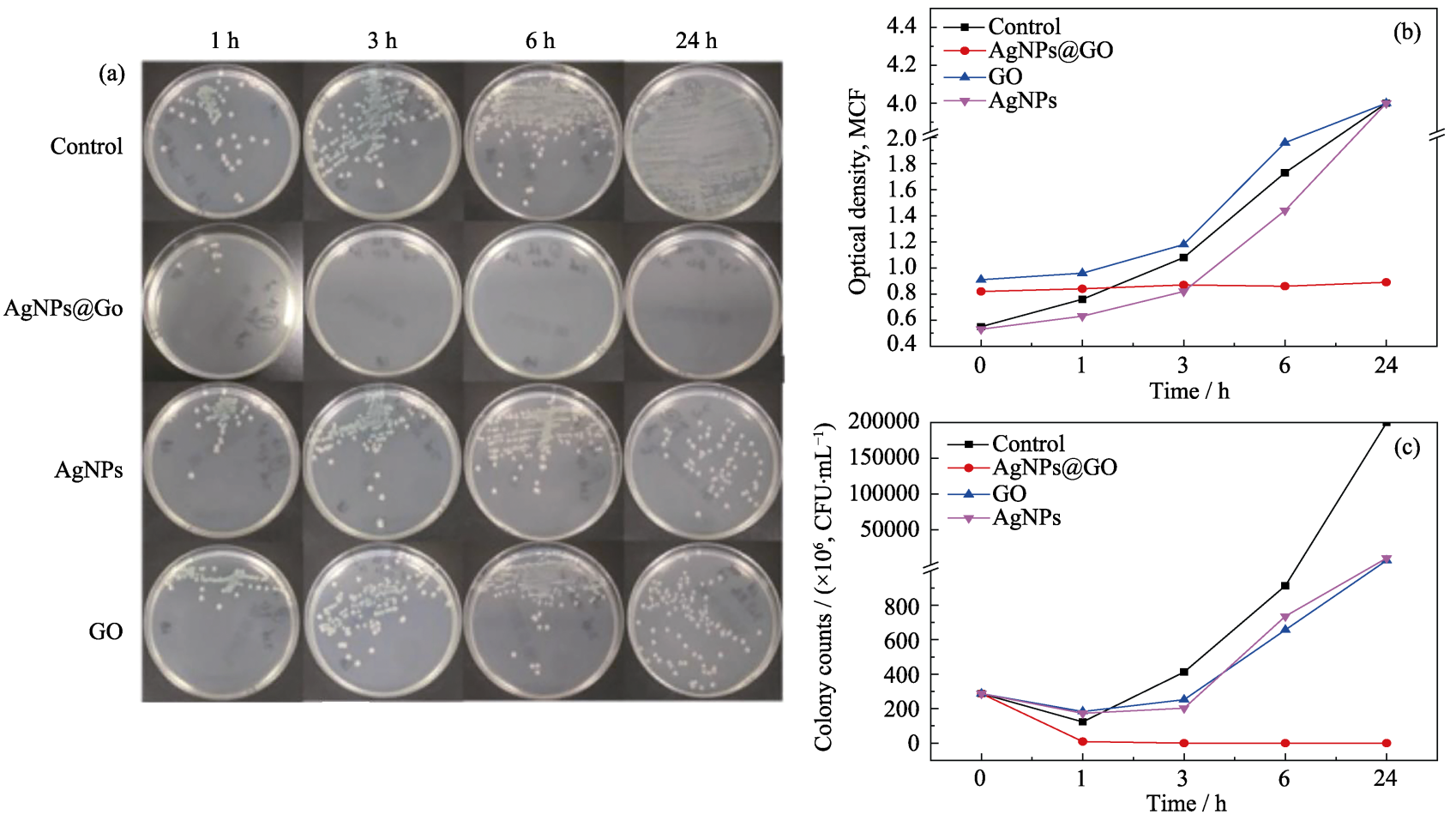

图 6 不同时间取菌液接种于营养琼脂平板上进行的细菌培养照片(a)及其在不同 时间的各试管中菌液的浊度(测量上限为 $4 \mathrm{MCF})(\mathrm{b})$ 和计数曲线(c)

Fig. 6 Photos (a) and turbidity (b) of the bacteria liquid in each test tube at different time (The upper limit of turbidity measurement is $4 \mathrm{MCF}$ ) and its counting curves of the bacterial culture on the nutrient agar plate at different time (c)

果均表明 AgNPs@GO 能有效抑制铜绿假单胞菌的 生长进而有可能切断传播途径, 并且其抑菌能力明 显优于 AgNPs 和 $\mathrm{GO}$ 。

\section{$2.30 .48 w t \%-A g N P s @ G O / P E$ 的抗菌性能}

如图 7 所示, 在 1、3、6、48 和 $72 \mathrm{~h}$ 各个时间 点抑菌能力强弱顺序为: 0.48wt\%-AgNPs@GO/PE > $2.88 \mathrm{wt} \%-\mathrm{AgNPs} / \mathrm{PE}>0.48 \mathrm{wt} \%-\mathrm{AgNPs} / \mathrm{PE}>$ 空白对 照组。其中掺杂低浓度 $(0.48 w t \%)$ 的 AgNPs@GO 的
材料 $0.48 \mathrm{wt} \%-\mathrm{AgNPs} @ \mathrm{GO} / \mathrm{PE}$ 显示了最强的抑菌能 力, 掺杂高浓度 $(2.88 \mathrm{wt} \%) \mathrm{AgNPs}$ 的材料 $2.88 \mathrm{wt} \%$ $\mathrm{AgNPs} / \mathrm{PE}$ 虽然比掺杂低浓度 $(0.48 \mathrm{wt} \%) \mathrm{AgNPs}$ 的 $0.48 \mathrm{wt} \%-\mathrm{AgNPs} / \mathrm{PE}$ 材料的抗菌能力强, 但仍然弱 于 $0.48 \mathrm{wt} \%$-AgNPs@GO/PE。

推测 $\mathrm{AgNPs} @ \mathrm{GO}$ 能把 $\mathrm{AgNPs}^{[23-27]}$ 与 $\mathrm{GO}^{[28]}$ 的 抗菌能力叠加起来, 并且产生了协同效应, 使 AgNPs@GO 的抗菌能力大幅增强。另外, 由于 $\mathrm{GO}$ 


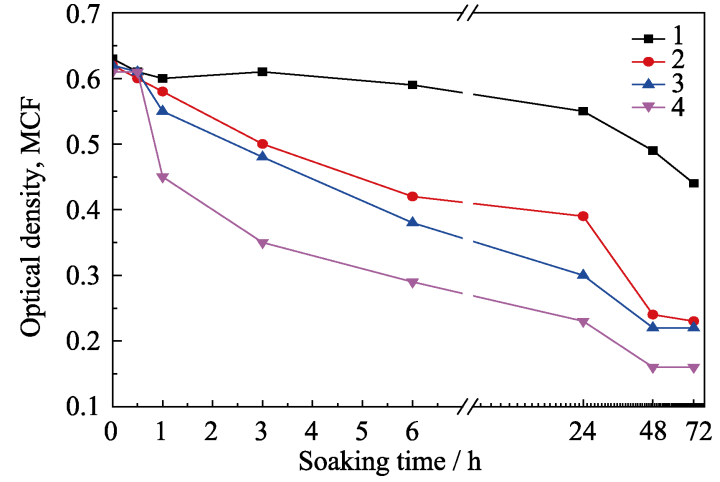

图 7 抑制铜绿假单胞菌生长实验结果

Fig. 7 Inhibition of Pseudomonas aeruginosa growth test results

Line 1: Blank control group; Line 2: $0.48 \mathrm{wt} \%-\mathrm{AgNPs} / \mathrm{PE}$; Line 3: 2.88wt\%-AgNPs/PE; Line 4: 0.48wt\%-AgNPs@GO/PE

表面大量的含氧基团能将 $\mathrm{AgNPs}$ 固定在片层结构 上, GO 对 AgNPs 起到了稳定和保护作用, 减少了 AgNPs 的溶出, 从而降低了纳米银的细胞毒性, 提 高了AgNPs@GO 以及 $0.48 \mathrm{wt} \%-\mathrm{AgNPs} @ \mathrm{GO} / \mathrm{PE}$ 的 长效抗菌能力。

\section{$2.40 .48 w t \%-A g N P s @ G O / P E$ 的阻隔水蒸气 性能}

如图 8 所示, 在标准测试条件下 $0.48 \mathrm{wt} \%$ $\mathrm{AgNPs} / \mathrm{PE}$ 薄片 (图 S5) 的水蒸气透过率为 $0.07477 \mathrm{~g} /\left(\mathrm{m}^{2} \cdot \mathrm{d}\right)$ 高于 $\mathrm{PE}$ 薄片(图 $\mathrm{S} 4$ )的水蒸气透过 率为 $0.03885 \mathrm{~g} /\left(\mathrm{m}^{2} \cdot \mathrm{d}\right)$, 而 $2.88 \mathrm{wt} \%-\mathrm{AgNPs} / \mathrm{PE}$ 薄片 (图 S6)的水蒸气透过率更高, 为 $0.09484 \mathrm{~g} /\left(\mathrm{m}^{2} \cdot \mathrm{d}\right.$ ), 这说明随着掺入的 $\mathrm{AgNPs}$ 的增加，水蒸气透过率有 上升趋势。然而 $0.48 \mathrm{wt} \%-\mathrm{AgNPs} @ \mathrm{GO} / \mathrm{PE}$ 薄片(图 S7) 的水蒸气透过率仅为 $0.02410 \mathrm{~g} /\left(\mathrm{m}^{2} \cdot \mathrm{d}\right)$, 比 PE 薄 片下降了 $38.5 \%$, 这说明 AgNPs@GO 的掺入能有 效地提高 PE 基复合材料阻隔水蒸气的性能。这一 现象一方面可能是由于 $\mathrm{AgNPs} @ \mathrm{GO}$ 上丰富的极性 基团能在复合材料内部形成氢键, 减缓了水蒸气

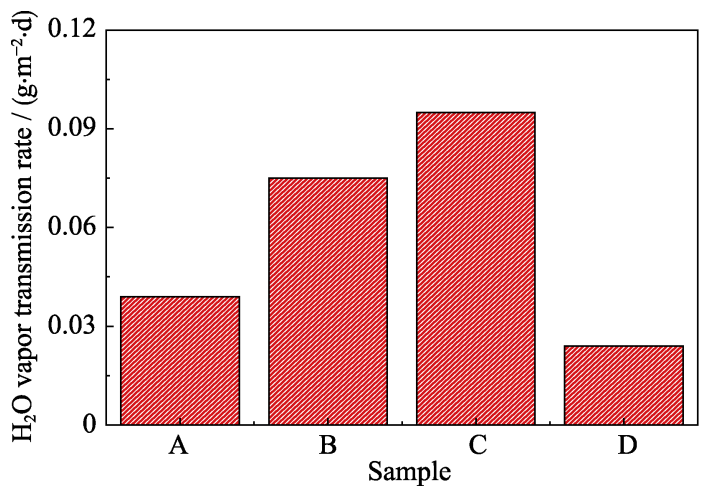

图 8 水蒸气透过率实验结果

Fig. 8 The results of water vapor transmission rate experiments A: PE; B: $0.48 \mathrm{wt} \%-\mathrm{AgNPs} / \mathrm{PE}$; C: $2.88 \mathrm{wt} \%-\mathrm{AgNPs} / \mathrm{PE}$; D: $0.48 \mathrm{wt} \%-$ AgNPs@GO/PE

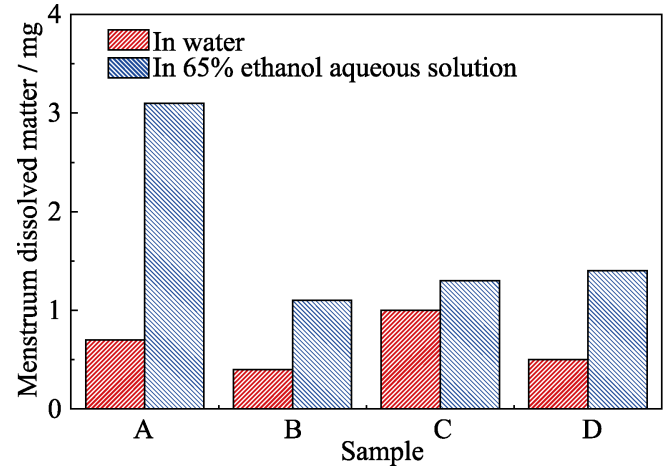

图 9 溶出物实验结果

Fig. 9 The results of the dissolution tests

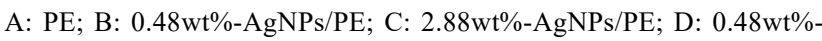
$\mathrm{AgNPs} @ \mathrm{GO} / \mathrm{PE}$

分子在复合材料内部的扩散速度，另一方面也可归 因于部分 AgNPs@GO 的二维片层结构取向与薄片 平行, 能很好地阻隔水分子的扩散传递。

\section{$2.50 .48 w t \%-A g N P s @ G O / P E$ 的耐溶出性能}

如图 9 所示, 0.48wt\%-Ag@GO/PE 薄片在 $121{ }^{\circ} \mathrm{C}$ 高压水浴 $30 \mathrm{~min}$ 溶出的不挥发物质量为 $0.5 \mathrm{mg}$, 略 小于相同条件下 PE 薄片的不挥发物溶出量 $0.7 \mathrm{mg}$, 但 $2.88 \mathrm{wt} \%-\mathrm{AgNPs} / \mathrm{PE}$ 在高压水浴中的不挥发物溶 出量为 $1.0 \mathrm{mg}$ 明显高于 PE 薄片。此外, $0.48 \mathrm{wt} \%$ $\mathrm{Ag} @ \mathrm{GO} / \mathrm{PE}$ 薄片、0.48wt $\%-\mathrm{AgNPs} / \mathrm{PE}$ 薄片和 $2.88 \mathrm{wt} \%-\mathrm{AgNPs} / \mathrm{PE}$ 薄片浸泡在 $58{ }^{\circ} \mathrm{C}$ 的 $65 \%$ 乙醇溶 液中 $30 \mathrm{~min}$ 溶出的不挥发物量分别为 $1.4 、 1.1$ 和 $1.3 \mathrm{mg}$ ，均远小于相同条件下 PE 薄片不挥发物溶出 量 $3.1 \mathrm{mg}$, 这说明 $0.48 \mathrm{wt} \%-\mathrm{Ag} @ \mathrm{GO} / \mathrm{PE}$ 在水和乙醇 溶液中都具有较好的耐溶出性能。

\section{3 结论}

采用绿色简便的超声波辅助液相法制备了一种 新的负载纳米银的氧化石墨烯材料 AgNPs@GO, 该材料能有效抑制铜绿假单胞菌生长, 并且其抑菌 性能优于 AgNPs 和 GO 材料。AgNPs@GO 掺杂 PE 制备的新型复合材料 $0.48 \mathrm{wt} \%-\mathrm{AgNPs} @ \mathrm{GO} / \mathrm{PE}$ 具有 更好的抑菌能力和更强的阻隔水蒸气性能, 并且在 水和乙醇溶液中都显示出较好的耐溶出性能, 有望 用于食品和药品的包装材料。

\section{参考文献}

[1] ZAHRA S A, BUTT Y N, NASAR S, et al. Food packaging in perspective of microbial activity: a review. J. Microbiol. Biotech. Food Sci., 2018, 6(2): 752-757.

[2] LIM M, KIM D, SEO J. Enhanced oxygen-barrier and waterresistance properties of poly(vinyl alcohol) blended with poly(acrylic acid) for packaging applications. Polym. Int., 2016, 
65(4): 400-406.

[3] JJOKAR M, RAHMAN R A, IBRAHIM N A, et al. Melt production and antimicrobial efficiency of low-density polyethylene (LDPE)-silver nanocomposite film. Food Bioprocess Tech., 2012, 5(2): 719-728.

[4] DAgLiA M. Polyphenols as antimicrobial agents. Curr. Opin. Biotechnol., 2012, 23(2): 174-181.

[5] KIM J S, KUK E, YU K N, et al. Antimicrobial effects of silver nanoparticles. Nanomed-Nanotechnol., 2007, 3(1): 95-101.

[6] SANCHEZ-VALDES S. Sonochemical deposition of silver nanoparticles on linear low density polyethylene/cyclo olefin copolymer blend films. Polym. Bull., 2014, 71(7): 1611-1624.

[7] LUO CHEN, DONG ZHENG, LI ZHEN-XING, et al. The effect of nano-silver antibacterial package on the quality of shrimp meat during cold storage. Packging Engineering, 2018, 39(7): 60-64.

[8] HE Y, QIAN L, LIU X, et al. Graphene oxide as an antimicrobial agent can extend the vase life of cut flowers. Nano Res., 2018: $1-13$.

[9] GEORGAKILAS V, TIWARI J N, KEMP K C, et al. Noncovalent functionalization of graphene and graphene oxide for energy materials, biosensing, catalytic, and biomedical applications. Chem. Rev., 2016, 116(9): 5464-5519.

[10] PHAM T A, KUMAR N A, JEONG Y T. Covalent functionalization of graphene oxide with polyglycerol and their use as templates for anchoring magnetic nanoparticles. Synthetic Met., 2010, 160(17/18): 2028-2036.

[11] DU W, LÜ Y, LU H, et al. Surface modification by graphene oxide: an efficient strategy to improve the performance of activated carbon based supercapacitors. Chinese Chem. Lett., 2017, 28(12): 2285-2289.

[12] JI H, SUN H, QU X. Antibacterial applications of graphene-based nanomaterials: recent achievements and challenges. Adv. Drug Deliver. Rev., 2016, 105: 176-189.

[13] THAVANATHAN J, HUANG N M, THONG K L. Colorimetric detection of DNA hybridization based on a dual platform of gold nanoparticles and graphene oxide. Biosens. Bioelectron., 2014, 55(10): 91-98.

[14] RODRIGUEZ-GONZÁLEZ C, VELAZQUEZ-VILLALBA P, SALAS P, et al. Green synthesis of nanosilver-decorated graphene oxide sheets. Iet Nanobiotechnol., 2016, 10(5): 301-307.

[15] DU W, LÜ Y, CAI Z, et al. Flexible all-solid-state supercapacitor based on three-dimensional porous graphene/titanium-containing copolymer composite film. Acta Phy.-Chim. Sin., 2017, 33(9): 1828-1837.

[16] 姜兴茂,闵建中,黎珊. 制备单分散超细颗粒的方法：中国, CN104690295A. 2013. 12. 05.

[17] LU C, MAI Y W. Preparation, characterization and antibacterial properties of silver-modified graphene oxide. J. Mater. Chem., 2011, 21(10): 3350-3352.

[18] MOULDER J F, STICKLE W F, SOBOL P E, et al. Handbook of X-ray Photoelectron Spectroscopy. Japan: ULVAC-PHI, Inc, 1992: $12-86$.

[19] TANG J, CHEN Q, XU L, et al. Graphene oxide-silver nanocomposite as a highly effective antibacterial agent with species-specific mechanisms. ACS Appl. Mater. Interfaces, 2013, 5(9): 3867-3874.

[20] DAS M R, SARMA R K, SAIKIA R, et al. Synthesis of silver nanoparticles in an aqueous suspension of graphene oxide sheets and its antimicrobial activity. Colloid. Surface. B, 2011, 83(1): 16-22.

[21] MESARO N, NORDMANN P, PLESIAT P, et al. Pseudomonas aeruginosa: resistance and therapeutic options at the turn of the new millennium. Clin. Microbiol. Infect., 2007, 13(6): 560-578.

[22] POLLACK M, YOUNG L. Protective activity of antibodies to exotoxin A and lipopolysaccharide at the onset of Pseudomonas aeruginosa septicemia in man, J. Clin. Invest., 1979, 63(2): 276-286.

[23] SONG H, KO K, OH I, et al. Fabrication of silver nanoparticles and their antimicrobial mechanisms. Eur. Cells. Mater., 2006, 11: 58.

[24] FENG Q L, WU J, CHEN G Q, et al. A mechanistic study of the antibacterial effect of silver ions on Escherichia coli and Staphylococcus aureus. J. Biomed. Mater. Res., 2000, 52(4): 662-668.

[25] SONDI I, SALOPEK-SONDI B. Silver nanoparticles as antimicrobial agent: a case study on $E$. coli as a model for Gram-negative bacteria. Colloid Interface Sci., 2004, 275(1): 177-182.

[26] LIU Z, STOUT J E, BOLDIN M, et al. Intermittent use of coppersilver ionization for Legionella control in water distribution systems: a potential option in buildings housing individuals at low risk of infection. Clin. Infect. Dis., 1998, 26(1): 138-140.

[27] PARK H J, KIM J Y, KIM J, et al. Silver-ion-mediated reactive oxygen species generation affecting bactericidal activity. Water Res., 2009, 43(4): 1027-1032.

[28] ZHENG H, MA R, GAO M, et al. Antibacterial applications of graphene oxides: structure-activity relationships, molecular initiating events and biosafety. Sci. Bull., 2018, 63(2): 133-142. 
补充信息

\title{
基于纳米银负载氧化石墨烯的新型聚乙烯复合材料
}

\author{
张 峰 ${ }^{1,2}$, 张凯立 ${ }^{1}$, 周明明 ${ }^{2}$, 陈 超 $^{3}$, 蔡志威 ${ }^{3}$, 魏国辉 ${ }^{4}$, \\ 姜兴茂 ${ }^{4,5}$, 张 诚 ${ }^{1}$, 劳伦 . 鲁尔曼 ${ }^{6}$, 吕耀康 ${ }^{1}$
}

(1. 浙江工业大学 化学工程学院, 杭州 $310014 ; 2$. 浙江大学医学院附属儿童医院, 杭州 310052 ; 3. 浙江省食品药 品检验研究院，杭州 310052；4. 常州英中纳米科技有限公司，常州 213000；5. 武汉工程大学化工与制药学院， 武汉 430205; 6. 斯特拉斯堡大学 化学研究所, 斯特拉斯堡 67081)

\section{1. 氧化石墨烯水分散液的制备}

称取 $1 \mathrm{~g}$ 的鳞片石墨, 置于 $100 \mathrm{~mL}$ 烧杯中, 依 次加 $12 \mathrm{~mL}$ 浓硫酸, $5 \mathrm{~g}$ 过硫酸钾, $5 \mathrm{~g}$ 五氧化二磷。 将混合物置于 $80{ }^{\circ} \mathrm{C}$ 油浴加热搅拌反应 $6 \mathrm{~h}$ 。反应结 束后，用大量去离子水抽滤洗涤上述混合物，直至 混合物的 $\mathrm{pH} \approx 7$, 再将其置于 $60{ }^{\circ} \mathrm{C}$ 真空烘干, 得到 预氧化石墨。第二步将预氧化石墨置于 $250 \mathrm{~mL}$ 烧 杯中, 加入 $0.8 \mathrm{~g}$ 硝酸钠, $34 \mathrm{~mL}$ 浓硫酸, 将烧杯置于 冰水浴中, 边搅拌边缓慢加入 $5 \mathrm{~g}$ 高锰酸钾。然后, 将烧杯置于 $40{ }^{\circ} \mathrm{C}$ (精确控温)水浴中摚拌反应 $2 \mathrm{~h}$ 。 将反应后所得的混合物再放入冰水浴中, 边搅拌边 分别加入 $100 \mathrm{~mL}$ 去离子水与 $4 \mathrm{~mL}$ 双氧水 $(30 \%)$, 得 到橙黄色的混合液。将所得到的混合液用大量去离 子水通过离心分离的方法进行若干次洗涤, 直至离 心上层清液 $\mathrm{pH} \approx 7$ 。将离心下层沉淀的氧化石墨取 出与一定量去离子水混合, 超声剥离 $3 \mathrm{~h}$ 。之后将混 合液通过 $6000 \mathrm{r} / \mathrm{min}$ 离心 $6 \mathrm{~min}$, 分离出未完全剥离 的石墨, 取上层液体, 即为氧化石墨烯(GO)水分散 液。再将氧化石墨烯 $(\mathrm{GO})$ 水分散液使用冷冻干燥得 到氧化石墨烯 $(\mathrm{GO})$ 。

\section{2. 其他实验材料}

鳞片石墨，型号： 325 mesh, 99.9995\%，购自阿 法埃莎(中国)化学有限公司; 低密度聚乙烯, 型号: $2426 \mathrm{H}$, 购自神华化工有限公司, 密度为 $0.925 \mathrm{~g} / \mathrm{cm}^{3}$, 熔体流动速率为 $2.16 \mathrm{~g} / 10 \mathrm{~min}$; 浓硫酸 $(95 \%$ 98\%), 购自西陇科学股份有限公司; 硝酸钠 $\left(\mathrm{NaNO}_{3}, \geqslant\right.$ $99 \% \mathrm{AR})$ 、五氧化二磷 $\left(\mathrm{P}_{2} \mathrm{O}_{5}, \geqslant 98 \% \mathrm{AR}\right)$ 均购自阿拉 丁试剂 (上海) 有限公司; 高锰酸钾 $\left(\mathrm{KMnO}_{4}, \geqslant\right.$ $99.5 \%, \mathrm{AR}$ )购自国药集团化学试剂有限公司; 纳米 银溶胶由常州英中纳米科技有限公司提供。

\section{AgNPs@GO 的结构与性质表征}

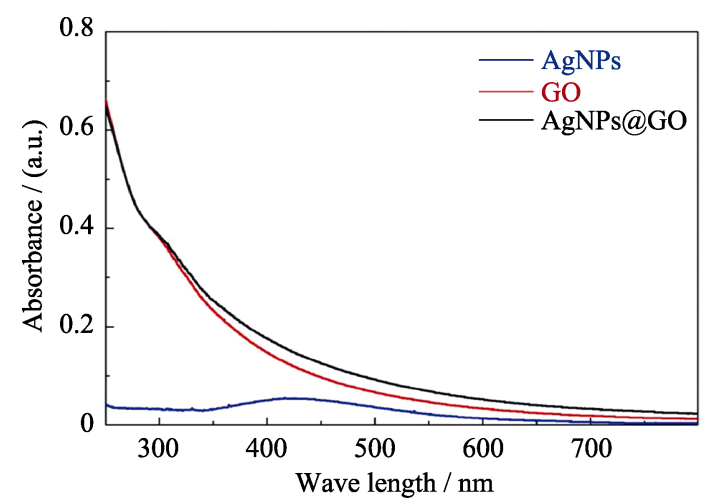

图 S1 AgNPs、GO 和 AgNPs@GO 水分散液的紫外-可见 吸收光谱

Fig. S1 UV-Vis spectra of AgNPs、GO and AgNPs@GO aqueous dispersions

\section{Debye-Scherrer 公式计算 $\mathrm{X}$ 射线粉末衍射} 数据

$$
D=\frac{K \gamma}{B \cos \theta}
$$

采用 Debye-Scherrer 公式(1)估算晶粒尺度,

其中 $K$ 为 Scherrer 常数 $K=0.89$,

$\theta$ 为衍射角, 选取的衍射峰 $2 \theta=37.980^{\circ}$,

$B$ 为实测样品衍射峰半高宽度 (FWHM) 为 $0.341^{\circ}$,

$\gamma$ 为 $\mathrm{X}$ 射线波长, 实验使用的是铜靶，因此 $\gamma=$ $0.154056 \mathrm{~nm}$,

通过公式(1)计算得晶粒尺寸 $D$ 约为 $24.36 \mathrm{~nm}$ 。

\section{5. 复合材料薄片的红外光谱分析}

如图 S3 所示, PE、0.48wt\%-AgNPs/PE、 $2.88 \mathrm{wt} \%-\mathrm{AgNPs} / \mathrm{PE}$ 和 $0.48 \mathrm{wt} \%-\mathrm{AgNPs} @ \mathrm{GO} / \mathrm{PE}$ 具 有相似的红外光谱, 位于 $2915.59 \mathrm{~cm}^{-1}$ 处的吸收峰 
对应的是- $\mathrm{CH}_{2}$-的不对称伸缩振动峰, $2847.87 \mathrm{~cm}^{-1}$ 处的吸收峰对应的是- $\mathrm{CH}_{2}$-的对称伸缩振动峰, 而 $1467 \mathrm{~cm}^{-1}$ 处的吸收峰是 $-\mathrm{CH}_{2}$-的弯曲振动峰, 同时 位于 $725 \mathrm{~cm}^{-1}$ 处的吸收峰是- $-\left(\mathrm{CH}_{2}\right)_{\mathrm{n}}-(\mathrm{n} \geqslant 4)$ 的面内 摇摆振动峰。1262.19 $\mathrm{cm}^{-1}$ 处的吸收峰是反式 $-\mathrm{CH}=\mathrm{CH}_{2}$ 的面外变形峰; $1099.86 \mathrm{~cm}^{-1}$ 处的吸收峰 是 $-\mathrm{CH}=\mathrm{CH}_{2}$ 的面外变形峰; $1023.82 \mathrm{~cm}^{-1}$ 处的吸收 峰是 $-\mathrm{CH}=\mathrm{CH}_{2}$ 的面外变形峰; $800.86 \mathrm{~cm}^{-1}$ 处的吸收 峰是 $>\mathrm{C}=\mathrm{CH}_{2}$ 的面外变形峰。通过红外光谱可以验 证所使用的 $\mathrm{PE}$ 是低压高密度聚乙烯材料。

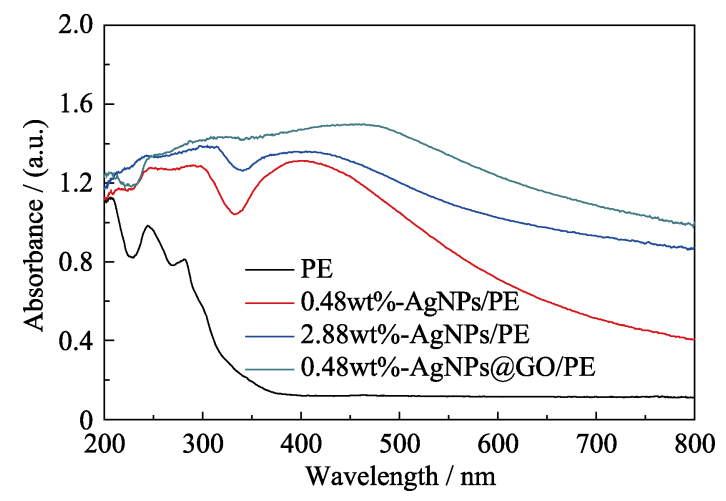

图 S2 PE、0.48wt \%-AgNPs/PE、 $2.88 w t \%-A g N P s / P E$ 和 $0.48 w t \%-A g N P s @ G O / P E$ 的紫外-可见吸收光谱

Fig. S2 UV-Vis spectra of PE, 0.48wt\%-AgNPs/PE, 2.88wt\% -AgNPs/PE, and 0.48wt\%-AgNPs@GO/PE

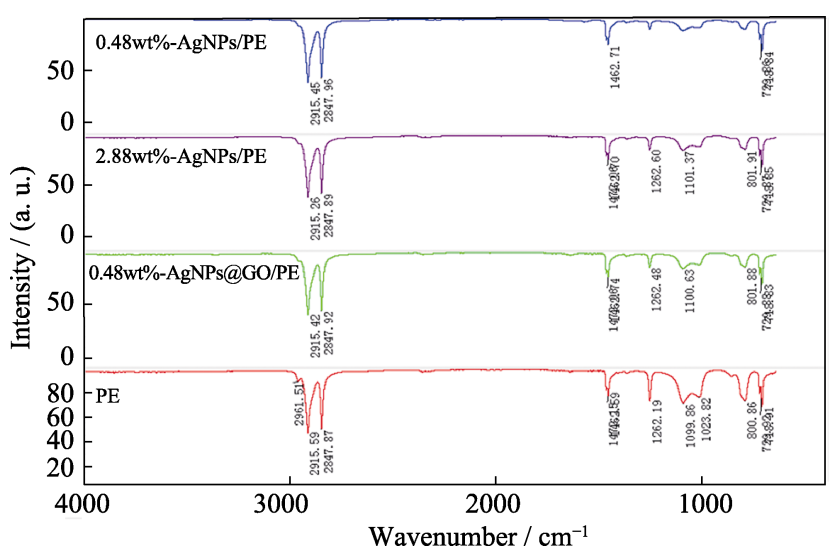

图 S3 PE、0.48wt \%-AgNPs/PE、 $2.88 w t \%-A g N P s / P E$ 和 $0.48 w t \%-A g N P s @ G O / P E$ 的红外光谱

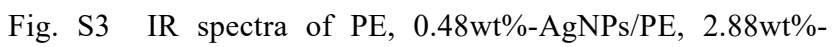
AgNPs/PE, and 0.48wt\%-AgNPs@GO/PE

\section{6. 复合材料薄片的紫外-可见吸收光谱分析}

图 S2 分别为 PE、0.48wt\%-AgNPs/PE、2.88wt\%$\mathrm{AgNPs} / \mathrm{PE}$ 和 $0.48 \mathrm{wt} \%$-AgNPs@GO/PE 薄片在 200 $800 \mathrm{~nm}$ 波长范围内的紫外一可见吸收光谱。

由图可见， $0.48 \mathrm{wt} \%-\mathrm{AgNPs} / \mathrm{PE} 、 2.88 \mathrm{wt} \%$ $\mathrm{AgNPs} / \mathrm{PE}$ 在 $430 \mathrm{~nm}$ 附近均具有明显的吸收峰, 归
属为 $\mathrm{Ag}$ 单质的特征吸收峰。表明了纳米银是以单 质形式存在复合材料中。0.48wt $\%-A g N P s @ G O / P E$ 的紫外-可见吸收光谱与 $0.48 \mathrm{wt} \%-\mathrm{AgNPs} / \mathrm{PE}$ 相比有 明显的不同, 在 250 550 nm 处有更宽的吸收带, 这 可以归因于 GO 的吸收带。

\section{7. 溶出物测试实验}

取内表面积 $50 \mathrm{~cm}^{2}$ 切成 $3 \mathrm{~cm} \times 0.3 \mathrm{~cm}$ 小块, 加 水 $100 \mathrm{~mL}$, 置于 $121{ }^{\circ} \mathrm{C}$ 高压灭菌锅内 $30 \mathrm{~min}$ 取出, 另制 1 份分别加 $65 \%$ 乙醇溶液 $100 \mathrm{~mL}$, 在 $58{ }^{\circ} \mathrm{C}$ 的 恒温水浴中保温 $2 \mathrm{~h}$ 。分别量取水、 $65 \%$ 乙醇与对应 空白试液 $100 \mathrm{~mL}$, 置于已恒重的蒸发血中水浴蒸干, 在 $105{ }^{\circ} \mathrm{C}$ 下干燥至恒重。

\section{8. 水蒸气透过率的测量}

根据塑料薄膜和薄片水蒸气透过率测定的国家 标准 GB/T 21529-2008, 水蒸气透过率数据采集自 美国 MOCON 公司 MCON Pematran-W3/33 水蒸气 透过率测试仪。测试条件为: 环境温度为 $23.0{ }^{\circ} \mathrm{C}$, 压 力为 $760 \mathrm{mmHg}$, 相对湿度为 $91.2 \%$, 水蒸气流速为 $9.77 \mathrm{sccm}$ 。

\section{MODULE INFORMATION: \\ Module 1, Serial \\ Setup Name: \\ Temp Setpoint/Actual: \\ Barometric Pressure: \\ Relative Humidity: \\ Flow Rate: \\ Ambient Temp:}

$\mathrm{M+} 01073$

5905532225Default Setup

Auto: $23.0 / 23.0^{\circ} \mathrm{C}$.

Manual: $760.00 \mathrm{~mm} \mathrm{Hg}$

Cell B - Auto: $91.2 \%$

Auto: 9.77 SCCM

Manual: $23.0^{\circ} \mathrm{C}$.

\section{CELL B INFORMATION:}

$\begin{array}{ll}\text { Sample Type: } & \text { Film: } 50 \mathrm{~cm} 2,1 \text { mil } \\ \text { Test Mode: } & \text { Continuous } \\ \text { Control Params: } & \text { Infinite } \\ \text { Exam Minutes: } & 30 \\ \text { Individual Zero: } & \text { No Ind. Zero } \\ \text { Conditioning: } & 0.5 \text { Hours } \\ \text { Cycles Complete: } & 30 \\ \text { Current Status: } & \text { Testing } \\ \text { Started Testing: } & 12 / 18 / 2017 \text { 9:14:22 AM } \\ \text { Elapsed Time: } & 23: 00\end{array}$

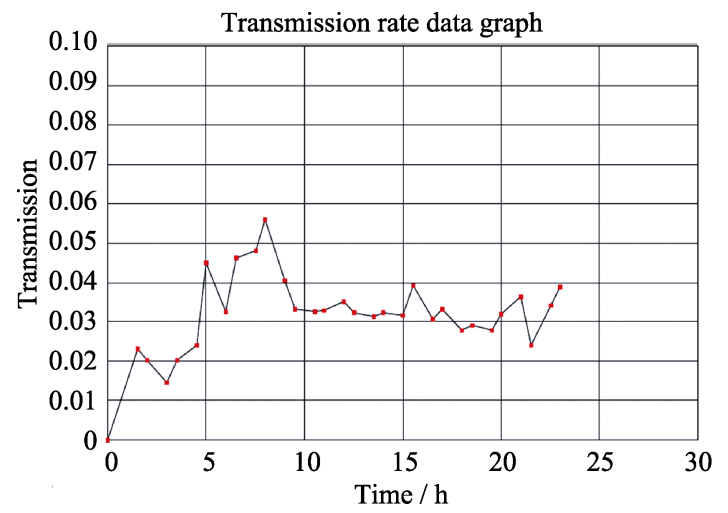

图 S4 PE 薄片的水蒸气透过率数据图, 其水蒸气透过率为 $0.03885 \mathrm{~g} /\left(\mathrm{m}^{2} \cdot \mathrm{d}\right)$

Fig. S4 Water vapor transmittance data of PE sheet is $0.03885 \mathrm{~g} /\left(\mathrm{m}^{2} \cdot \mathrm{d}\right)$ 


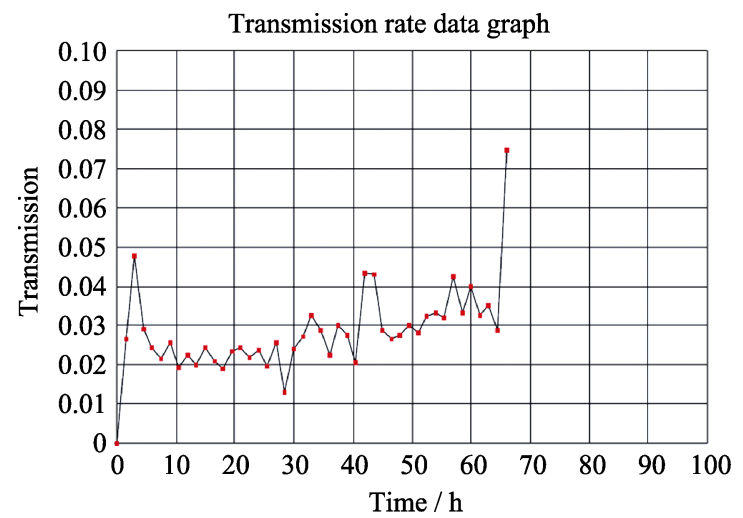

图 S5 $0.48 \mathrm{wt} \%-\mathrm{AgNPs} / \mathrm{PE}$ 薄片的水蒸气透过率数据图, 其水蒸气透过率为 $0.07477 \mathrm{~g} /\left(\mathrm{m}^{2} \cdot \mathrm{d}\right)$

Fig. S5 Water vapor transmittance data of $0.48 \mathrm{wt} \%-A g N P s / P E$ sheet is $0.07477 \mathrm{~g} /\left(\mathrm{m}^{2} \cdot \mathrm{d}\right)$

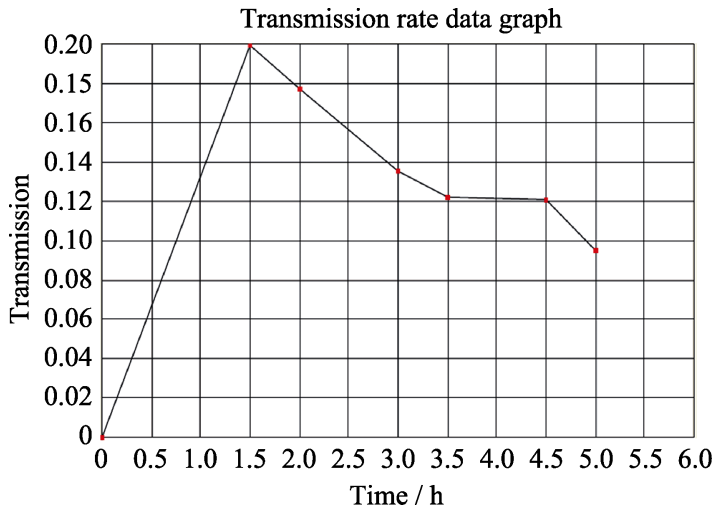

图 S6 2.88wt\%-AgNPs/PE 薄片的水蒸气透过率数据图，其水蒸气透过率为 $0.09484 \mathrm{~g} /\left(\mathrm{m}^{2} \cdot \mathrm{d}\right)$

Fig. S6 Water vapor transmittance data of $2.88 \mathrm{wt} \%-\mathrm{AgNPs} / \mathrm{PE}$ sheet, $0.09484 \mathrm{~g} /\left(\mathrm{m}^{2} \cdot \mathrm{d}\right)$

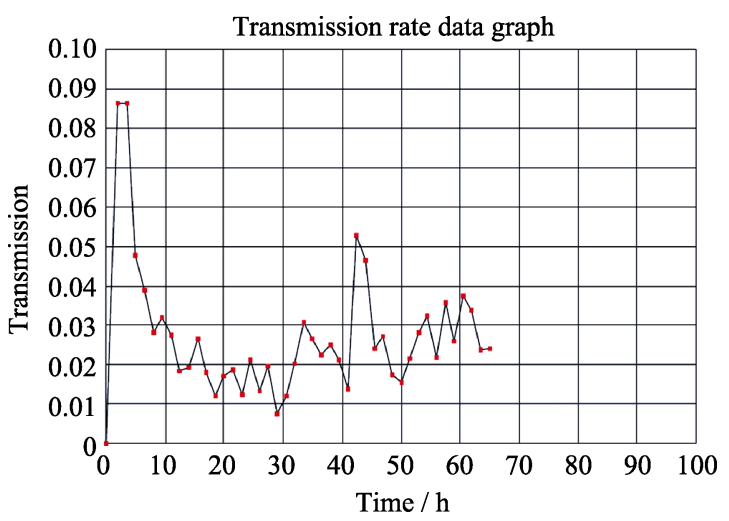

图 S7 $0.48 \mathrm{wt} \%-\mathrm{AgNPs} @ \mathrm{GO} / \mathrm{PE}$ 薄片的水蒸气透过率数据图, 其水蒸气透过率为 $0.02410 \mathrm{~g} /\left(\mathrm{m}^{2} \cdot \mathrm{d}\right)$

Fig. S7 Water vapor transmittance data of $0.48 \mathrm{wt} \%$-AgNPs@GO/PE sheet, $0.02410 \mathrm{~g} /\left(\mathrm{m}^{2} \cdot \mathrm{d}\right)$ 